\title{
Carnets
}

Revue électronique d'études françaises de l'APEF

Deuxième série - 13 | 2018

Corps, rythmes et voix : en/jeux littéraires et artistiques

\section{Lorsque la voix déchire la lettre}

La fiction au Moyen Âge comme poétique de l'entre-deux

\section{Carlos F. Clamote Carreto}

\section{(2) OpenEdition}

Journals

Édition électronique

URL : http://journals.openedition.org/carnets/2573

DOI : $10.4000 /$ carnets. 2573

ISSN : 1646-7698

Éditeur

APEF

Référence électronique

Carlos F. Clamote Carreto, « Lorsque la voix déchire la lettre », Carnets [En ligne], Deuxième série -

13 | 2018, mis en ligne le 31 mai 2018, consulté le 05 mai 2019. URL : http://journals.openedition.org/ carnets/2573; DOI : 10.4000/carnets.2573

Ce document a été généré automatiquement le 5 mai 2019.

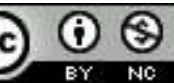

Carnets est mis à disposition selon les termes de la licence Creative Commons - Atribution - Pas d'utilisation commerciale 4.0 International. 


\title{
Lorsque la voix déchire la lettre
}

\author{
La fiction au Moyen Âge comme poétique de l'entre-deux
}

\author{
Carlos F. Clamote Carreto
}

Seignours, or fetes pes, franke gente honoree, Gardés k'il n'i ait noise ne corous ne mellee, S'orrés boné chanson de bien enluminee :

N'i sera fable dite ne mensonge provee. Niuls des altres jouglours, k'els le vous ont contee, Ne sevent de l'estoire vaillant une darree. La chanson ert perdue et la rime faussee ; Mais Gautier de Douay a la chaire membree Et li rois Lawis dont l'alme est trespassee (...) Par luy et par Gautier est l'estoire aünee

Et la chanchon descie, espeise et alumee, A Saint Dynis de France premerement trovee,

Del rolle de l'église escrite et translatee ; Cent ans i a esté, ch'est verité provee.

La Destruction de Rome, v. 1-15.

(...) dans la présence, la conscience sourde et pourtant aiguë d'un deuil ; dans l'espace insonore de l'écriture, la nostalgie poignante d'une voix humaine et vive; dans la pensée de l'absence, le regret incomblable d'une mesure éthique.

B. Clément (2012: 195) 


\section{Vox autem vivificat}

1 «Corps, rythmes et voix»: trois termes qui désignent cette union symbiotique d'où émerge la création littéraire sous fond d'un sacrifice primordial : celui qui se traduit par l'effacement du poète devant le balbutiement du cosmos et l'irruption incandescente des premières sonorités s'entrelaçant sur la couche nuptiale du rythme pour engendrer la langue dont la poésie est tissée. Eugénio de Andrade, Rosto precário (1995 : 19) :

É no mar crepuscular e materno da memória, onde as águas «superiores " não foram ainda separadas das "inferiores", que as imagens do poeta sonham pela primeira vez com a precária e fugidia luz da terra. Diante do papel, que «la blancheur défend", o poeta é uma longa e só hesitação. Que Ifigénia terá de sacrificar para que o vento propício se levante e as suas naves possam avistar os muros de Troia? Que augúrios escuta, que enigmas decifra naquele rumor de sangue em que se debruça cheio de aflição? Porque ao princípio é o ritmo; um ritmo surdo, espesso, do coração ou do cosmos - quem sabe onde um começa e o outro acaba? Desprendidas de não sei que limbo, as primeiras sílabas surgem, trémulas, inseguras, tacteando no escuro, como procurando um ténue, difícil amanhecer. Uma palavra de súbito brilha, e outra, e outra ainda. Como se umas às outras se chamassem, começam a aproximar-se, dóceis ; o ritmo é o seu leito ; ali se fundem num encontro nupcial, ou mal se tocam na troca de uma breve confidência, quando não se repelem, crispadas de ódio ou aversão, para regressarem à noite mais opaca.

2 Conservant la mémoire de cette rencontre primordiale, si la voix témoigne d'une plénitude symbolique, c'est également parce qu'elle relève d'un imaginaire et d'une ontologie fondée sur la Présence, son efficacité supposant qu'elle soit recueillie par une oreille bienveillante qui puisse l'intérioriser et ainsi la rendre fertile (Carreto, 2016). D'où ce lieu-commun des chansons de geste (mais le lieu-commun n'est-il pas justement cet espace privilégié de la re-connaissance structuré autour de topoi fonctionnant aussi bien comme puissante machina memorialis (Carruthers, 2002) qui renforce la cohésion de toute une communauté textuelle ${ }^{1}$ que comme lieu privilégié de réactivation de schèmes d'un imaginaire culturel et poétique commun) qui débute invariablement sur un appel au silence, l'entrée dans l'espace fictionnel (surtout lorsqu'il s'agit de cet espace solennel et quasi liturgique où l'on commémore les faits exemplaires des héros fondateurs) imposant ainsi une rupture face au désordre, au tumulte verbal, à la discorde (le principe de réalité, en somme) qui risquent de faire sombrer la voix poétique dans un chaos babélien situé aux antipodes de cette nouvelle Pentecôte du langage que l'émergence du récit désire réinstaurer. C'est également ce que nous rappelle Le Chevalier au Lion (Yvain) de Chrétien de Troyes qui s'ouvre sur le récit atypique à la première personne tenu par cet étrange anti-héros qu'est Calogrenant et dont les premiers vers (dirigés à l'auditoire) constituent une véritable poétique de la voix au creux de laquelle résonnent néanmoins de nombreuses notes dissonantes. La prise de la parole suppose certes toujours le retour à un silence matriciel et une écoute attentive à travers laquelle la voix - qui est transportée vers l'oreille comme "vens qui vole » - entreprend une trajectoire descendante jusqu'à être enclose par le ventre du cœur ${ }^{2}$. Cette parole est elle-même le fruit d'un long et douloureux processus de maturation, car elle éclot après sept longues années de silence durant la fête solennelle de la Pentecôte. Cependant, elle ne raconte pas une histoire d'amour comme toutes ces histoires, souligne Chrétien de Troyes, que dames, chevaliers et demoiselles partageaient alors à la cour; elle n'évoque pas non plus une aventure 
héroïque et exemplaire; elle ne parle pas d'honneur, mais de honte ${ }^{3}$ Le récit de Calogrenant est, en effet, une sorte de contre-récit : il est l'aveu d'un échec (l'épreuve ratée de la fontaine bouillante à l'entrée du royaume de Laudine) qui - chose surprenante au regard de tous ceux qui assistent à cette étrange confession ${ }^{4}$ - s'accompli en absence du roi Arthur qui, ce jour-là, après avoir été retenu par la reine dans sa chambre, a perdu la notion du temps et s'est endormi ! L'histoire racontée par Calogrenant et la pulsion à la fois destructrice, expiatoire et rédemptrice qui l'accompagne semble ainsi se situer paradoxalement en marge de l'autorité, devenant ainsi une voix suspecte, voir illicite, qui trouble et parasite le logos arthurien. Mais le surgissement scandaleux de cette voix ne coïncide pas seulement avec l'effacement de l'autorité royale: elle semble également impliquer une mise en retrait de l'autorité poétique. Remarquons en effet que Le Chevalier au Lion est le seul des cinq romans de Chrétien de Troyes où le prologue n'assume pas clairement une dimension métapoétique ${ }^{5}$. Cette dimension, peut-on rétorquer, est partiellement transférée sur le discours de Calogrenant sous forme de mise en abîme fictionnelle. Elle ne témoigne pas moins d'un distancement ambigu de l'instance auctoriale qui semble devoir s'éclipser face au pouvoir troublant de la voix; un effacement qui n'est d'ailleurs pas sans rappeler, à l'autre bout du récit et de l'autre côté du miroir textuel, l'image elliptiquement esquissée d'une pucelle qui, dans le verger où va se dérouler la dernière et la pire des aventures d'Yvain, lit à ses parents un roman «ne sai de cui $»^{6}$, affirme le narrateur, c'est-à-dire un roman dont la paternité est elle aussi mystérieusement tue ou fourvoyée.

3 Ces observations initiales nous invitent d'ores et déjà à relativiser les couples latins vs roman, oral vs écrit durant longtemps présentés comme antithétiques au Moyen Âge. En effet, non seulement ces deux couples ne se recouvrent-ils pas exactement ${ }^{7}$, comme ils fonctionnent suivant une dynamique complexe et paradoxale tissée à la fois de complicités et de rivalités latentes. Si l'émergence du roman (comme langue et discours poétique) au Moyen Âge témoigne bien d'une émancipation face aux modèles latins et néo-latins qui implique une prise de conscience de la singulière dynamique scripturaire agissant au cœur de la fiction ${ }^{8}$, les réflexions menées dans les années 80 par Paul Zumthor (1987) et Bernard Cerquiglini (1989) ont clairement montré que cette littérature naissante ne suppose nullement une rupture face à la matrice orale sur laquelle elle se fonde (avec tout ce que cela implique d'un point de vue mytho-poétique) et qui ne cesse de traverser et de déchirer la lettre. À la fois archaïque et obstinément présente à travers la performance orale ${ }^{9}$, la voix qui retentit dans les œuvres du Moyen Âge est mystérieuse, complexe et plurielle: elle ressoude constamment les fractures du temps et renoue inlassablement les liens distendus entre l'écriture et le mythe. Séductrice, elle exerce une envoutante puissance d'attraction en même temps qu'elle divise et sépare ${ }^{10}$, relevant ainsi à la fois du symbolique et du diabolique.

4 Une lecture attentive des prologues narratifs (tous genres confondus: chansons de gestes ou de croisade, vies de saints, romans courtois, antique et bretons, récits didactiques et allégoriques, etc.) met clairement en évidence que l'oralité (ou, du moins, ces mises en scène fictionnelles de l'oralité qui nous intéressent ici tout particulièrement) n'est aucunement une instance homogène et parfaitement délimitée ni du point de vue sémantique et formel ni du point de vue structural. Ces prologues s'ouvrent d'ailleurs très souvent sur une topique bien connue (mais là encore, il faut prendre les lieux-communs au sérieux!) qui oppose une oralité positive à une oralité négative dessinant les contours d'une rivalité au seuil de l'antithèse entre deux figures majeures de la transmission 
poétique au Moyen Âge : le poète/troubadour et le jongleur. Commençons par la part maudite de l'oralité. Quelle que soit la stratégie rhétorique adoptée, tous les poèmes s'accordent sur un point: il faut exorciser à tout prix le spectre de la corruption/ dégradation de la parole poétique dont les jongleurs sont les principaux chantres. Nous trouvons déjà ce célèbre topos dans le premier roman de Chrétien de Troyes, Érec et Énide (éd. 1981), et il se répète invariablement tout au long la tradition littéraire du Moyen Âge ${ }^{11}$ :

D'Erec, le fil Lac, est li contes,

Que devant rois et devant contes

Depecier et corronpre suelent

Cil qui de conter vivre vuelent.

Des or comancerai l'estoire

Qui toz jorz mes iert an mimoire

Tant con durra crestïantez;

De ce s'est Crestïens vantez (v. 19-26).

5 Le jongleur malfamé - image au miroir négative du poète dont l'ombre menace obsessionnellement l'écriture - incarne tous les vices capitaux sur le plan moral tout comme poétique, dans la mesure où sa version du récit s'ancre dans une chaîne de transmission purement orale qui suppose la lacune, l'oubli, la transformation intéressée (apologétique et souvent associée au désir du gain) de la matière ${ }^{12}$, le déplacement de village en village, de cour en cour, semblant introduire une fragmentation qui se projette sur une performance poétique qui fait du récit une sorte de manteau mal taillé1 ${ }^{13}$, décousu, déformé, adultère et éthiquement réprouvable :

Aprentiç jougleour et escrivain mari,

Qui l'on de lieus en lieus ça et la conqueilli,

Ont l'estoire fausse, onques mais ne vi si

(Adenet Le Roi, Berte as grans piés, v. 13-15).

6 Ces injonctions contre les jongleurs suggèrent sans doute que la légitimité du fait littéraire ne saurait plus reposer uniquement, à partir du XII siècle, sur l'autorité vocal, impliquant de plus en plus une épistémologie de l'écrit dont le texte exhume les vestiges réels ou purement fictionnels. D'où cet art de la pseudographie, véritable «mirage des sources » (Dragonetti, 1987), que le Moyen Âge a su cultiver comme nul autre à travers l'image particulièrement suggestive $\mathrm{du}$ livre enfoui dans la bibliothèque d'une prestigieuse abbaye (Saint-Martin de Tour, Saint-Pierre de Beauvais, Saint-Denis, etc. ${ }^{14}$ ) où le poète déclare avoir trouvé (verbe poétique par excellence) le manuscrit (latin) dont le récit serait la translatio directe en roman.

7 Fruit du hasard (comme dans Bertes aux grands pieds d'Adenet le Roi) ou d'un cheminement laborieux, la découverte du livre traduit une sorte de retour vers la terre promise où la matière narrative se révèle dans son intégrité et unicité originelles, lieu où le Sens incarne. L'espace (le topos) du livre est certes un non-lieu qui nous lance au seuil de l'aporie ${ }^{15}$. Il montre néanmoins à quel point l'absence peut être signifiante du point de vu symbolique et à quel point l'imaginaire de la Présence peut émerger sur fond d'un vide fondateur. Cette fiction légitimatrice du livre source enfoui dans l'espace (con)sacré de l'abbaye ou du monastère, de l'écrit comme auctoritas et paternité pré-textuelle sans laquelle nulle parole nouvelle ne peut advenir, n'est pas d'ailleurs sans évoquer la figure des griots qui, bien que déclamant de mémoire, «montrent l'ouvrage fermé à terre qui attesterait, s'il était consulté, qu'il contient bien l'histoire qu'ils narrent et qu'eux-mêmes n'en changent pas le moindre mot » (Payen, 1990 : 172). Ce livre enfoui sous la terre que le 
griot tout à la fois vénère, invoque et foule des pieds ou celui que l'on découvre initiatiquement dans les recoins d'une bibliothèque monastique, participe bien entendu de la topique de la clôture et du secret, une des innombrables variations sur thème traditionnel du trésor caché comme hypostase du Sens. Mais il se rattache également à toute une symbolique de la régénération.

\section{Pour une grammaire de la voix}

Bien que cela soit tentant, il serait alors réducteur d'interpréter la présence légitimatrice du livre au sein de tous ces récits comme le signe indéniable d'une rupture entre l'oral et l'écrit et d'une subordination de la voix à l'empire tout-puissant de la lettre. Suggérant une épistémologie centrée sur la phonè, la voix est souffle, mémoire d'une parole archaïque et lointaine ${ }^{16}$, que l'écriture à un certain moment fixe et immobilise. Outre l'important aspect mémoriel, cette fixation par l'écriture produit un double effet de sens contraire : il donne une forme à la matière et au sens sans laquelle le récit serait pure virtualité vocale. De nombreux poèmes insistent ainsi sur la dimension quasi rituelle et initiatique qui préside à la fabrication et à la transmission du livre où la matière poétique sera gardée et scellée :

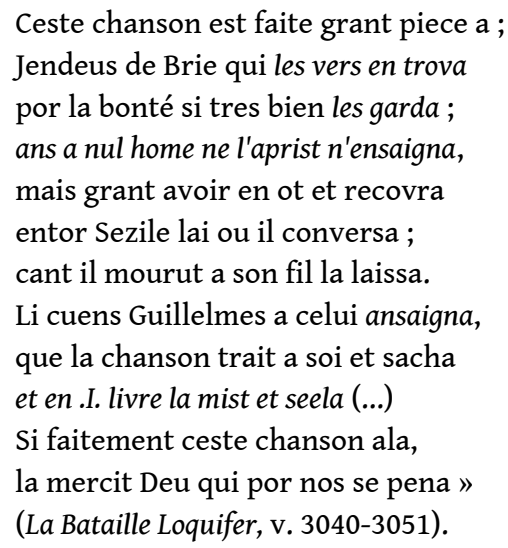

9 Face aux aléas de la transmission orale, l'écrit s'érige en principe de réunification d'une matière diverse, fragmentaire et éparse ${ }^{17}$. Si la voix est pulsion déviante (songeons à nouveau à Calogrenant), errance, menace de dispersion, l'écriture vient restaurer l'intégrité d'une parole archaïque, renouer les liens distendue ou perdus, retrouver un archi-texte (un Urtext) qui n'a jamais existé mais auquel l'image du livre vient donner corps :

Qui d'Aleschans out les vers controvez, et tous ces moz perduz et oubliez; ne sot tant qu'il les eüst rimez. Or les vous a Guilaume restorez, cil de Batpaumes, qui tant est bien usez de chançons fere et de vers acesmez (Le Moniage Rainouart, texte du ms A, v. 7462.1-6). ${ }^{18}$

Mais la nature foncièrement conservatrice de l'écriture risque de cristalliser la lettre et d'en pétrifier mortellement le sens. Il faut donc qu'à nouveau un souffle vocal vienne revivifier la lettre (en la traduisant en roman, par exemple, pour mieux la disséminer), faire vibrer et résonner son rythme et ébranler l'écrit afin d'en délivrer pleinement le sens ${ }^{19}$ : 
La gentil geste doit on renoveler

(Mort Aymeri de Narbonne, v. 16).

Si comme fu en romans translatee

Et par un clerc nos fu renouvelee

(Beuve de Hamptone, v. 14-15)

Mais Garinsdor de Douai nel velt mie oblier

Ki nos en a les vers fais renoveler

(Chanson d'Antioche, v. 14-15).

11 Pour ne pas corrompre la matière du conte comme le font les jongleurs sans scrupules, cette voix doit cependant procéder directement de la source écrite, évitant tout hiatus spatial, linguistique et temporel -, toute discontinuité qui porterait atteinte à son intégrité. Émerge alors une image lumineuse de la voix poétique qui repose à la fois sur une éthique ${ }^{20}$ et sur une paradoxale grammaire (au sens étymologique du terme ${ }^{21}$ ) de l'oralité.

12 Face au déclin de la chanson de geste ${ }^{22}$ à travers la parole corrompue de la fable jougralesque, le récit vrai se décline alors invariablement sous le signe de la rectitude. Dans son prologue de Méraugis de Portlesguez, Raoul de Houdenc oppose clairement son « nouveau conte » (v. 19) à celui des autres faiseurs de rimes qui, n'ayant pas étudié profondément la matière, ne sont que des "contrediseurs" (v. 13) dont les mots se révèlent totalement vides de sens. Le Roman du roi Yder va encore plus loin en séparant radicalement l'univers de la fable mensongère de celui de la vérité de l'estoire et en proscrivant de surcroît l'inflation hyperbolique du réel comme emblème d'une rhétorique fictionnelle du faux :

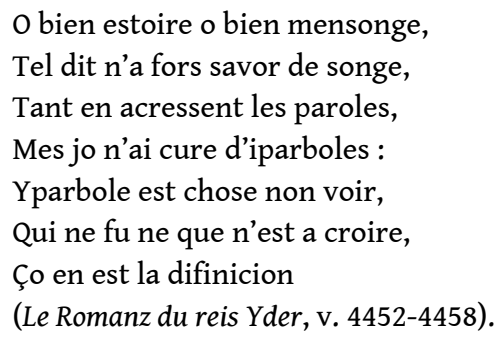

13 Si l'invention poétique consiste à (re)trouver (trover, troveure, trovement en ancien français) et à restaurer l'intégrité, la cohérence et l'homogénéité d'une parole ancestrale perdue ${ }^{23}$ ou ensevelie, oubliée, déformée ou gardée dans le plus profond des secrets, son double négatif (la fable) se place, quant à lui, toujours sous le signe de la controvure où la mimésis devient contrefaçon aux contours lucifériens ${ }^{24}$ :

Ce sachiez, n'est pas controvure,

Ainz est tot estrait d'escriture ${ }^{25}$.

A Tors ou mostier sain Martin

Le trovai escrit en latin

(Robert de Blois, Biaudouz, v. 283-286).

Qui d'Aliscans ot les viers controvés

Ot tous ces mos perdus et desvoiés.

Ore les ra Guillaumes restorés (...)

(Alicans, v. 37-38).

Rétablir le lien entre l'ordre du discours et l'ordre des choses (isomorphe, dans la pensée symbolique du Moyen Âge, de l'ordre de l'univers) d'où émane le récit vrai exige, par conséquent, un acte de restauration, voire de correction, de la grammaire du conte dont 
la syntaxe et la musicalité (rythme, rime, etc.) doivent impérativement se fonder sur les principes de rectitude, de mesure et d'équilibre, miroirs de la concordia du monde et de la Création:

Nus hom ne puet chançon de jeste dire

Que il ne mente la ou li vers define,

As mos drecier et a tailler la rime.

Ce est biens voirs, gramaire le devise,

Uns hom la fist de l'anciene vie,

Hues ot non, si la mist en livre

Et seela el mostier Saint Denise

La ou les jestes de France sont escrites

(La Mort Aymeri de Narbonne, v.

3055-3062).

15 Face au pouvoir séducteur mais déformant de la parure rhétorique (métaphores, hyperboles, etc.) qui portent atteinte à la propriété intrinsèque des mots et des choses, cette grammaire de la fiction se décline alors suivant les contours d'une singulière géométrie rectiligne :

Pour ce qu'est mal rimee, la rime amenderai

Si a droit que l'estoire de riens ne fausserai,

Mençonge ne oiseuse ja n'i ajouterai

Mais parmi la matere droite voie en irai

(Adenet le Roi, Buevon de

Conmarchis, v. 21-24). ${ }^{26}$

Corriger, remanier, réordonner et redresser cette fable discordante et dissonante transmise par les jongleurs devient ainsi un impératif à la fois éthique et poétique pour accorder discours, sens et raison au sein d'un récit dont l'énonciation, placé sous l'égide d'une rigoureuse arithmétique du vers, rejoint dès lors la vaste et harmonieuse syntaxe de l'univers (la «mesure du monde» dont parlait Paul Zumthor [1993] dans un de ses derniers essais). C'est ce que souligne, de façon particulièrement éloquente, le prologue des Enfances Ogier d'Adenet le Roi :

Cil jougleor qui ne sorent rimer,

Ne firent force fors que dou tans passer,

L'estoire firent en plusours lieus fausser,

D'amours et d'armes et d'onnour mesurer

Ne sorent pas les poins compasser,

Ne les paroles a leur droit enarmer

Qui apartienent a noblement diter ;

Car qui ceste estoire ver par rime ordener,

Il doit son sens a mesure acorder

Et a raison, sans poin de descorder (v. 13-22) ${ }^{27}$

\section{La voix transfuge}

17 Mais quel crédit accordé aux déclarations véridictionnelles de tous ces poètes dont le discours évoque bien souvent le paradoxe du menteur? Cherchant à tout prix à exorciser le spectre de la fable mensongère, tous se réclament de la vérité de l'estoire ${ }^{28}$ où le langage poétique se définit nostalgiquement comme retour à l'union (ou du mois à la contigüité) sacramentelle et utopique entre les mots et les choses ${ }^{29}$. C'est ainsi qu'une œuvre comme le Roman de Troie de Benoît de Sainte-Maure se permettra même de contester l'autorité d'Homère et de démentir sa version du siège de Troie, déclarant qu'il va translater en 
roman celle qui fut premièrement traduite en latin par Cornélius à partir de «l'estoire que Daire [Darès de Phrygie] ot escrite / en grecque langue faite et dite » (v. 91-92), car ce dernier - né et élevé à Troie - avait témoigné de ses propres yeux (v. 106) les évènements qu'il a immédiatement retranscrits la nuit suivante ${ }^{30}$. Mais si Benoît de Sainte-Maure semble ici proposer clairement un idéal poétique reposant sur une translatio rectiligne et ininterrompue à partir de la source latine retrouvée (le roman comme translittération), comment concilier cette idéal avec le principe toujours suspect de la controveüre (généralement liée, comme nous l'avons signalé plus haut, à une transmission - orale qui déforme la matière et fragmente le sens) que le poète revendique orgueilleusement comme étant à l'origine de sa création?

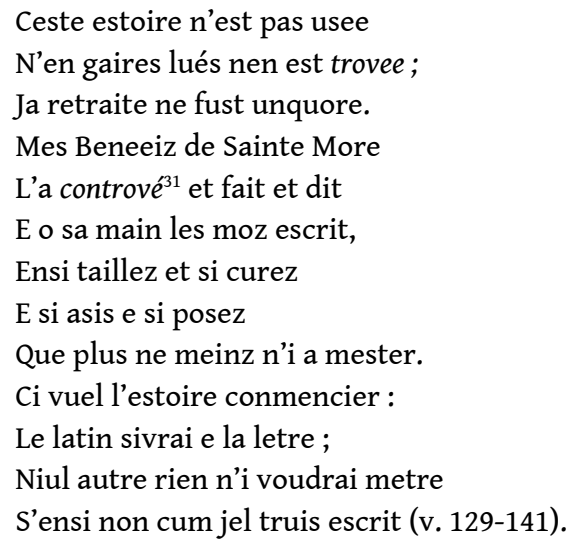

Que penser également de ce singulier et paradoxal roman du $\mathrm{xII}^{\mathrm{e}}$ siècle, Guillaume d'Angleterre, qui, sous l'apparence du noble registre hagiographique (modèle de la vie de saint Eustache), déploie en fait le troublant récit d'un roi qui devient marchand et d'une reine amnésique et adultère tentée par le désir de dévorer ses propres enfants pour rassasier son incontrôlable faim. Cette étrangeté n'est pas seulement narrative ou sémantique : elle s'inscrit au sein même de l'ordre poétique à travers une stupéfiante contradiction entre l'idéal de rectitude par rapport à la source livresque affiché par le prologue $^{32}$ et l'épilogue où le poète déclare, sans qu'aucune contrainte narrative ne l'y oblige, avoir puisé son récit dans une source purement orale dont l'autorité est pour le moins suspecte ou défaillante ${ }^{33}$ :

Tele est de ce conte la fins,
Plus n'an sai, ne plus n'an i a.
La matiere si me conta
Uns miens compeins, Rogiers li cointes,
Qui de meins preudome iert acointes.

Explicit la vie seint Guillaume qui fu rois d'Angleterre (v. 3102-3106).

19 Le développement d'une conscience littéraire en langue vulgaire n'est donc ni simple affirmation d'une présence vocale qui résonne au creux de l'écriture, ni pure revendication du pouvoir de la lettre face à une oralité dont le prestige symbolique commencerait à s'émietter à partir de la seconde moitié du XII siècle. Elle engage toujours une dynamique complexe des rapports entre la lettre et la voix, l'écrit, le rythme et le corps. Le roman de Perlesvaus (ou le Haut Livre du Graal - début du XIII ${ }^{\mathrm{e}}$ siècle), par exemple, confère certes à Joseph le statut de l'auctor élu qui reçoit et met par écrit les paroles sacrées dictées par la voix de l'Ange. L'espace qui abrite le Livre est toujours, certes, celui de l'abbaye, mais cet espace ne prétend plus désormais engendrer un effet de réel, la géographie qu'il déploie devenant celle, purement autoréférentielle, de la fiction qui 
acquiert ainsi une autonomie presque scandaleuse, au seuil de la profanation. La fin du Perlesvaus nous révèle en effet que ce livre latin où puise le roman est trouvé dans une sainte abbaye située à la limite des Marais Aventureux dans lîle d'Avalon où reposent le roi Arthur et la reine Guenièvre et où repose également - étrange coïncidence - le Graal lui-même, du moins d'après Le Roman de l'Estoire du Graal de Robert de Boron. L'espace du livre est donc transposé dans l'Autre-Monde intangible et troublant de la légende arthurienne dont il scelle la mort, le seul témoin palpable de la tradition se réduisant désormais à cette inlassable voix qui retransmet sans fin la légende.

Remplaçant la voix de l'ange dans Perlesvaus, c'est Merlin qui, dans le roman homonyme de Robert de Boron assume le rôle d'auctor-dictator démiurgique qui rétablit les maillons manquant dans la chaîne symbolique du langage et du monde, Blaise se rangeant au statut du copiste élu qui recueille fidèlement les révélations du prophète dans l'espace matériel d'un Livre dont l'unité est scellée («ansis sera tes livres celez », 16, 102-103) 34 $^{34}$ par l'imposition d'un Nom - le titre : « si avra non toz jorz mais (...) tes livres li Livres dou Graal », 23, 62-64 - qui assure la droite paternité de l'écriture ${ }^{35}$. Tout semble parfait, à condition que l'on oublie, par exemple, que la voix d'où émerge le Livre est fatalement imprégnée de l'origine duelle de Merlin, le fils du diable racheté par Dieu. À condition que l'on oublie également que le mage du roi Arthur n'est pas le témoin direct de tous les faits qu'il dicte : il se souvient, mais n'a pas vécu le temps de l'invention du Graal (le roman Joseph qui constitue le premier livre) et Blaise, quant à lui, il n'a même connu le monde arthurien. Or, c'est Merlin lui-même qui prend le soin de nous rappeler qu'il ne peut y avoir autorité sans témoignage personnel, d'où l'on conclue que ce Livre ne possède qu'un faible statut auctorial ${ }^{36}$, aussi suspect et problématique que la voix de ce personnage qui lui donne corps.

21 Le roman n'est pas le seul genre poétique perméable au distancement ironique par rapport aux origines doubles et obscurs du récit. Le Moniage Guillaume, chanson de geste du XII ${ }^{e}$ siècle, témoigne d'une même instabilité de la source. En effet, alors que le prologue déploie les éléments traditionnels de la topique du Livre ( De fiere geste bien sont les vers assis ;/ N'est pas juglerres qui ne set de cestui ;/ L'estoire en est au mostier Saint Denis,/ Molt a lonc tens qu'ele est mise en oubli ;/ Molt fu preudom cil qui rimer la fist » (v. 3-7), voici qu'au moment où le narrateur s'apprête à raconter la rencontre cruciale entre Guillaume d'Orange et l'ermite Gaidon, surgit un troublant «ce conte li romanz » (v. 2149) qui brise totalement l'image de la source latine, ancestrale et immuable, gardée au monastère, une image qui ne sera reprise qu'à la fin de la chanson («si con lisant trovon ", v. 6856) pour une raison stratégique, car elle devra coïncider avec le moment où l'histoire du héros-saint doit s'élever au rang du mythe fondateur.

Ni même l'hagiographie, genre pourtant prestigieux et au registre si grave, n'échappe véritablement au désœuvrement produit par une voix transfuge qui se plait à mettre constamment en déroute une grammaire trop rigide et figée du récit. La Vie de saint Thomas Becket (circa 1174) attribuée à Guernes de Pont-Sainte-Maxence s'ouvre par un long prologue (165 vers) qui parodie clairement la topique de la source livresque trouvée dans une abbaye (celle de Canterbury, en l'occurrence). Le récit traduit en roman se présente ici comme le fruit d'une complexe et douloureuse besogne ${ }^{37}$ qui consiste à mettre en confrontation les diverses leçons orales du conte, à les comparer à la source écrite (latine) de la vie du saint et à soumettre la version définitive à un minutieux travail sur la forme (rimes, rythme, etc.) qui n'est pas donné à tout le monde et sans lequel nul récit se saurait devenir chant poétique : 
Tuit li fysicien ne sunt adès bon mire ;

Tuit clerc ne sevent pas bien chanter ne dire ;

Asquanz des troveürs faillent a bien dire (v. 1-3). sérieux, par ailleurs), la première version du récit composé par le poète devenant un objet si grand de désir et de convoitise qu'elle est volée (« cel premier romanz m’uns escrivein emblé », v. 151) avant même d'avoir été corrigée et améliorée, finissant par être vendue et achetée par un « riche umme » (v. 158). Voici un prologue aux contours métapoétiques assez original dans la mesure où il remplace le motif habituel du conte déformé par la voix corrompue et corruptrice du jongleur par l'image d'une translatio manuscrite dévoyée à la fois par la mercantilisation du récit sous l'effet du désir mimétique et par la circulation d'une contrefaçon qui transforme la pseudo-vie de saint Thomas Becket («cil autre romanz ke unt fait ", v. 161) en une fausse monnaie du sens où la vérité côtoie le mensonge ${ }^{38}$. Comme on pourra s'en douter, cet égarement n'est qu'une ironique et subtile mise en scène rhétorique visant à mettre en valeur la supériorité de cette nouvelle parole poétique (la seconde version du roman) dont l'intégrité, la perfection, la mesure et l'harmonie musicale et langagière ne sauraient désormais que rimer avec le terme « vérité »:

Ainc mais si bons romans ne fu faiz ne trover.

A Cantorbire fu e faiz e amendez.

N'i a mis un sul mot qui ne seit veritez.

Li vers est d'une rime en cinc clauses cuplez.

Mis languages est bons, car en France fui nez (v. 6161-6165).

exples que nous venons de parcourir montrent clairement que les rapports entre la lettre et la voix ${ }^{39}$ ne suivent pas une hiérarchie parfaitement délimité et immuable. Outre une complexité historique qui provient du fait que le statut social, idéologique et culturel de l'oral face à l'écrit subit de nombreuses et profondes transformations surtout à partir du XII ${ }^{\mathrm{e}}$ siècle (transformations que le roman incarne et fait miroiter), les deux instances forment un doublet dynamique, voire dialogique, tissé de complicités, de paradoxes, d'asymétries et de rivalités plus ou moins discrètes ou ostensibles. Au cœur de cette dynamique, la Voix, ce «deuil de l'équivoque » selon la belle expression de Bernard Clément (2012 : 296), représente un souffle qui à la fois régénère l'écriture et la déchire en la confrontant sans cesse avec une altérité et une différence perturbatrice d'un ordre que la logique scripturaire aimerait imposer au récit. À l'époque où nait et se développe le récit fictionnel en langue vulgaire, ce rapport ambigu suggère-t-il peut-être une tension créatrice entre la prégnance symbolique qui émane de la voix et la dimension sémiotique incarnée par la lettre. Quoi qu'il en soit, il s'agit d'une tension qui, loin d'être fortuite ou conjoncturelle, est constamment recherchée, nourrie et relancée par les poètes, comme si la fiction narrative au Moyen Âge se définissait, par nature et vocation, comme une pensée et une expérience de l'entre-deux qui émerge du hiatus, de cette constante contrediction (Dragonetti, 1987 :51) entre la lettre et les pulsions profondes émanant de la voix dont les frontières instables, fluides et sans cesse reconfigurées dessinent une poétique de l'incertitude et de la discontinuité qui renvoient très souvent le lecteur au non-lieu d'une impossible confrontation. La voix ne serait-elle alors que cet éternel point de fuite par rapport à ce signifiant privilégié du désir qu'est la lettre au Moyen Âge ?

Carnets, Deuxième série - 13 | 2018 


\section{BIBLIOGRAPHIE}

ADENET LE ROI (1982). Berte as grans piés. Éd. critique A. Henry. Genève : Droz.

ADENET le RoI (1996). Buevon de Conmarchis. Éd. A. Henry, in Les CEuvres d'Adenet le Roi, Tome II. Genève : Slatkine.

ALICANS (2007). Éd. Cl. Régnier. Paris : Champion.

ANDRADE, EUGÉNIO de (1995). Rosto precário. Porto : Fundação Eugénio de Andrade.

BENOÎT DE SAINTE-MAURE (1998). Le Roman de Troie. Éd. bilingue E. Baumgartner et F. Vielliard. Paris : Le Livre de Poche, « Lettres Gothiques ».

BEUVE DE HAMPTONE (2014). Éd. bilingue de J.-P. Martin. Paris : Champion.

BLOCH, Howard (1986). The Scandal of the Fabliaux. Chicago: The University of Chicago Press.

BOUTET, Dominique (1993). La Chanson de geste. Forme et signification d'une écriture épique au Moyen Âge. Paris : PUF-Écriture.

BOUTET, Dominique (2006). « La voix : mirages et présence de l'oralité au Moyen Âge », in F. Lestringant et M. Zink, Histoire de la France littéraire, vol. I, Naissances, Renaissances, Moyen Âge-XVI ${ }^{e}$ siècle. Paris : PUF.

BOURGAIN, Pascale (2005). « Latin/langue vernaculaire », in J.-R. Valette (dir.). Perspectives médiévales. Trente ans de recherches en langues et en littératures médiévales. Paris : Société de Langues et de Littératures Médiévales d'Oc et d'Ö̈l.

CARRETo, Carlos F. CLAmote (2007). « Topique et utopie du livre au Moyen Âge : le texte (im)possible », in A. Milon et M. Perelman. Le Livre et ses espaces. Paris : Presses Universitaires de Paris 10, p. 35-61.

CARreto, Carlos F. clamote (2016). « A voz ou a plenitude do texto. Performance oral, práticas de leitura e identidade literária no Ocidente medieval », in Medievalista, 19 [Consulté le 13/10/2017]. <URL: http://www2.fcsh.unl.pt/iem/medievalista/MEDIEVALISTA19/carreto1903.html> CARRUTHERS, Mary (2002). Machina memorialis : Méditation, rhétorique et fabrication des images au Moyen Âge. Paris : Gallimard.

CERQUIGLINI, Bernard (1989). Éloge de la variante. Histoire critique de la philologie. Paris : Seuil. CERQUIGLINI-TOULET, Jacqueline (1992). La Couleur de la mélancolie. La fréquentation des livres au XIXe siècle-1300-1415. Paris : Hatier.

CHRÉTIEN DE TROYES (1981). Érec et Énide. Éd. M. Roques, Paris : Champion.

CHRÉTIEN DE TROYES (1994). Romans : Paris : Librairie Générale Française.

CLÉMENT, Bernard (2012). La Voix verticale. Paris : Belin.

DRAGONETTI, Roger (1987). Le Mirage des sources : l'art du faux dans le roman médiéval. Paris : Seuil. GAUTIER D'ARRAS (1988). Ile et Galeron. Éd. Y. Lefèvre. Paris : Champion. 
GUERNES DE PONT-SAINTE-MAXENCE (1936). La Vie de saint Thomas Becket. Éd. E. Walberg. Paris : Champion.

GUILLAUME D'ANGLETERRE (1988). Éd. critique A. J. Holden. Genève : Droz. ISIDORE DE SÉVILLE (1993). Etymologies. Éd. bilingue Latin/Espagnol de J. Oros Reta et M-A. Marcos Casquero. Madrid : Biblioteca de Autores Cristianos, vol. I.

KOBLE, Nathalie (2005). « Le Lai du cor » et « Le Manteau mal taillé ». Les dessous de la Table Ronde . Paris : Éditions rue d'Ulm.

LA BATAILLE LOQUIFER (1975). Éd. M. Barnett. Oxford: Blackwell, « Medium Aevum Monographs. New Series " 6 .

LA CHANSON D'ANTIOCHE (2011). Éd. B. Guidot. Paris : Champion.

LA DESTRUCTION DE ROME (1990). Éd. L. Formisano. Londres: Anglo-Norman Text Society, « Plain Texts Series ", 8.

LA MORT AYMERI DE NARBONNE (2000). Éd. critique P. Rinoldi. Milan : Unicopli.

LA PRISE D'ORANGE (1986). Éd. Cl. Régnier. Paris : Klincksieck.

LE MONIAGE GUillaume (2003). Éd. Critique N. Andrieux-Reix. Paris : Champion.

LE MONIAGE RAINOUART (1988). Éd. G. A. Bertin. Paris: Picard, vol. II.

LE ROMANZ DU REIS YDER (2010). Éd. critique et traduction par J. Ch. Lemaire. Fernelmont : E.M.E.

MERLIN (1979). Éd. A. Micha. Paris : Champion.

MÖLK, Ulrich. Les Débuts d'une théorie littéraire en France. Anthologie critique. Paris : Classiques

Garnier.

PAYEN, Jean-Charles (1990). Littérature française : le Moyen Âge. Paris : Arthaud.

RAOUl de houdenc (2014). Méraugis de Portlesguez. Éd. M. Szkilnik. Paris : Champion.

ROBERT DE BLOIS (2008). Biaudouz. Éd. critique et traduction par J.-Ch. Lemaire. Liège : Éditions de l'Université de Liège.

STоск, Brian (1983). The Implications of Literacy. Written Language and Models of Interpretation in the Eleventh and Twelfth Centuries. Princeton : Princeton University.

TRISTAN ET ISEUT : LES POÈMES FRANÇAIS - LA SAGA NORROISE (1989). Éd. Bilingue par Ph. Walter et D. Lacroix. Paris : Librairie Générale Française, coll. « Lettres Gothiques ».

VANDENDORPE, Christian (1999). Du papyrus à l'hypertexte. Essai sur les mutations du texte et de la lecture. Paris : La Découverte.

ZINK, Michel (1993). Introduction à la littérature française du Moyen Âge. Paris : Librairie Générale Française, « Le Livre de Poche » ZUMTHOR, Paul (1987). La Lettre et la voix. De la « littérature » médiévale. Paris : Seuil. ZUMTHOR, Paul (1993). La Mesure du monde : représentation de l'espace au Moyen Âge. Paris : Seuil.

\section{NOTES}

1. Sur cette notion, voir B. Stock (1983: 88-240). 
2. « (...) or entendés !/ Cuer et oroeilles me rendés,/ car parole oïe est perdue/ s'ele n'est de cuer entendue./ Or y a tix que che qu'il oent/ n'entendent pas, et si le loent;/ et chil n'en ont fors que l'oïe,/ puis que li cuers n'i entent mie ;/ as oreilles vient le parole,/ aussi comme li vens qui vole,/ mais n'i arreste ne demore,/ ains s'en part en mout petit d'ore,/ se li cuers n'est si estilliés/ c'a prendre soit appareilliés ;/ que chil le puet en son venir/ prendre et enclorre et retenir./ Les oreilles sont voie et dois/ ou par ent y entre la vois ;/ et li cuers prent dedens le ventre/ le vois qui par l'oreille y entre./ Et qui or me vaurra entendre,/ cuer et oreilles me doit rendre,/ car ne veul pas servir de songe,/ ne de fable, ne de menchonge » (v. 149-172 de l'éd. bilingue D. HUE, in Chrétien de Troyes [1994: 887]).

3. « (...) lor ot commenchié .i. conte,/ non de s'onnor, mais de sa honte» (v. 59-60).

4. « Mais chel jour mout s'esmerveillierent... » (v. 42).

5. Tous les autres prologues sont parcourus par des considérations d'ordre poétique ou rhétorique à travers lesquelles la conscience littéraire de Chrétien se manifeste très nettement : dans Érec et Énide, il nous explique l'originalité de sa technique de composition ancrée dans la « conjointure " des matières; dans Cligès il revendique clairement la paternité de ses œuvres, inscrivant son nom au sein d'une tradition littéraire dont il se veut à la fois l'origine rénovatrice et la fin (évocation d'une singulière translatio studii qui aboutit dans l'univers arthurien); dans Le Chevalier à la Charrette (Lancelot) et Le Conte du Graal (Perceval), Chrétien joue à cache-cache avec les destinataires/commanditaires de l'œuvre, créant de surprenants effets en «trompe-l'œil » autour des sources qu'il n'évoque que pour mieux les enfouir.

6. «Mesire Yvains el vergier entre/ Et aprés lui toute sa route ;/ Apuyé voit deseur son coute/ Un preudomme qui se gesoit/ Seur .i. drap de soie, et lisoit/ Une puchele devant li/ En un rommans, ne sai de cui./ Et pour le rommans escouter/ S'i estoit venue acouter/ Une dame, et estoit sa mere » (v. 5356-5365).

7. Affirmation qui fait de nos jours l'unanimité chez les médiévistes. Voir, par exemple, les considérations de M. Zink à ce sujet (1993: 14-19) ou celles de P. Bourgain (2005: 87-98) et de D. Boutet (2006: 193-225).

8. Voir l'intéressant ouvrage d'U. Mölk (2011).

9. Cette double dimension est parfaitement soulignée par B. Clément (2012:9) lorsqu'il affirme que la voix est «ce qui tient du langage et se souvient qu'elle touche à un temps qui ne le connaissait pas. »

10. C'est l'étymologie de seducere.

11. Girard de Roussillon (v. 4, 17), Jean Bodel, dans la Chanson des Saisnes (v. 25-28) vocifère contre ces «jongleurs bâtards » qui vont de village en villages et mettent en pièces la noble matière épique qu'ils transforment en pure fable; Anseïs de Carthage (v. 10-15) ; Adenet le Roi évoque, dans les Enfances Ogier, les jongleurs qui, ne dominant pas l'art de la rime, en viennent à fausser l'histoire (v. 13-19), Chanson d'Antioche (v. 12-13).

12. Voir D. Bouted (1993: 65-98).

13. Nous reconnaîtrons-là le titre d'un célèbre lai ou fabliau du XIII ${ }^{\mathrm{e}}$ siècle, Le Mantel mautaillé. Voir à ce sujet, les intéressantes réflexions d'H. Bloch (1986) et, plus récemment, celles de $\mathrm{N}$. Koble à son édition du texte (2005).

14. Je me limiterai à quelques exemples emblématiques: L'hagiographie en anglo-normand de Saint Thomas Becket de Guernes de Pont-Sainte-Maxence fait référence à l'Abbaye de Canterbury, Cligès de Chrétien de Troyes à Saint-Pierre de Beauvais (v. 21), le roman Guillaume d'Angleterre à l'Abbaye de Saint-Edmond (v. 15), le roman Beaudous de Robert de Blois au monastère de SaintMartin de Tours (v. 285). De tous ces lieux de culte qui étaient également au Moyen Âge, dans la plupart des cas, d'importants scriptoria, nous remarquerons une nette préférence pour l'Abbaye de Saint-Denis : La Destruction de Rome (v. 13), la Mort Aymeri de Narbonne (v. 3061), Doon de Mayence (v. 22), Girard de Vienne de Bertrand de Bar-sur-Aube (v. 8), Berte aus grans piés d'Adenet le Roi (v. 7), Les Enfances Guillaume (v. 5). Cela ne saurait être une pure coïncidence. En effet, l'espace de 
Saint-Denis ne représente-t-il pas le lieu par excellence où se réalise l'alliance entre le réel et le simulacre littéraire ? Où la légitimité du pouvoir temporel et religieux repose sur l'invention des pseudo-reliques de Denys l'Aréopagite ? Où la légitimité dynastique, cherchant à perpétuer la mémoire prestigieuse de l'héritage carolingien se fonde sur une reconstruction mythicolégendaire extrêmement élaborée de l'histoire de la France orchestrée, à ses débuts, par l'abbé Suger durant la première moitié du xiie siècle (la version latine des Grandes Chroniques de France) ? La seule évocation de ce signifiant nominal, lourd de résonnances idéologiques et imaginaires, à une époque où se développent les légendes épiques en langue vernaculaire, fonctionne, par conséquent, comme un double modèle. Un modèle positif, d'une part, dans la mesure où il permet d'ériger la fiction poetique au statue d'estoire et de l'ancrer dans un espace vénérable au sein duquel la parole poétique (à l'instar de la parole divine) est appelée à incarner dans le Livre. Modèle tout à la fois positif et négatif d'une mémoire textuelle bâtie sur l'art de la contrefaçon légitimatrice à laquelle aspire tout récit au Moyen Âge et en particulier le récit épique ou hagiographique.

15. Voir, à ce sujet, nos réflexions (Carreto, $2007: 35-61)$.

16. Voir, par exemple, des expression comme « estoire de vielle anchiserie » (Anseïs de Cathage, v. 5), «Bonne canchon (...) faite du tans ancienour » (Beuve de Hantone, v. 46), « de loing l'a aprise » ( Prise d'Orange, v. 19).

17. L'épilogue sur lequel se clôt le roman de Tristan de Thomas d'Angleterre (version du ms Douce) est probablement l'un des plus éloquent en ce qui concerne le problème auquel le poète se confronte lorsqu'il cherche à unifier par ses vers une matière trop diverse, contradictoire, voire incohérente : « Ne vol pas trop en uni dire :/ Ici diverse la matyre./ Entre ceus qui solent cunter/ E del cunte Tristran parler,/ Il en cuntent diversement :/ Oï en ai de plusur gent./ Asez sai que chescun en dit/ E ço qu'il unt mis en escrit,/ Mes sulun ço que j'ai oï,/ Nel dïent pas sulun Breri/ Ky solt les gestes e les cuntes/ De tuz les reis, de tuz les cuntes/ Ki orent esté en Bretaingne » (v. 840-852).

18. La Destruction de Rome va encore plus loin dans cette logique réparatrice en impliquant le roi Louis lui-même - devenu soudain troubadour - dans le processus scripturaire (voir le passage cité en exergue). Notons au passage que dans cet extrait l'écriture et la voix sont intimement liées à travers l'image du rouleau (le volumen) qui suppose une logique de lecture ancrée dans une temporalité linéaire et irréversible (par opposition au codex) comme le souligne à plusieurs reprises C. Vandendorpe (1999).

19. "Littera occidit, spiritus autem vivificat ", disait saint Paul dans son Épître aux Corinthiens qui sera amplement glosée par saint Augustin (De Doctrina Christiana, III, V, 9) afin de légitimer l'activité exégétique comme activité sacrée dans la mesure où qui permet de délivrer (au double sens de « libérer » et "transmettre ») le sens emprisonné dans la lettre, l'exégèse se présentant ainsi aux antipodes d'un rapport servile et idolâtre face à l'Écriture. Les récits profanes du Moyen Âge en langue vernaculaire gloseront très souvent ce thème (voir le prologue des Lais de Marie de France ou celui du Conte du Graal de Chrétien de Troyes, par exemple) pour faire la parole poétique une parole fertile qui régénère, à travers l'art de la reprise, de la réécriture, la lettre (morte) sur laquelle elle se fonde. D'où la Parabole du Semeur chez Chrétien de Troyes (Perceval), celle des Talents chez Marie de France, ainsi que l'emploi très fréquent de métaphores florale dans de nombreux prologues. Dans Bueve de Hantone, par exemple, les mauvais jongleurs sont ceux qui ont « oublié la flour » de l'estoire (v. 50) que le poète, grâce à sa voix, va faire refleurir.

20. Cette étique de la voix n'est pas sans évoquer la dimension « verticale » de la voix dont parle B. Clément (2012). En effet, indissolublement liée au corps et la présence d'autrui dans le hic et nunc de la performance orale, la voix a forcément partie liée au regard et au visage et, par conséquent, à la responsabilité - ou injonction - éthique qu'ils imposent, comme le rappelle Lévinas dans Éthique et Infini. 
21. C'est-à-dire, suivant la longue tradition rhétorique reprise par Isidore de Séville dans ses Etymologies (I, V, 1), la grammaire comprise comme "scientia recte loquendi, et origo et fundamentum liberalium litterarum. Haec in disciplinis post litteras communes inventa est, ut iam qui didicerant litteras per eam recte loquendi rationem sciant $"$.

22. Voir notamment l'incipit de La Mort Aymeri de Narbonne qui regrette «coment les jestes vindrent a decliner » (v. 4).

23. «La chanchon ert perdue et la rime fausée » (La Destruction de Rome, v. 7).

24. Voir J. Cerquiglini-Toulet (1992: 107).

25. Remarquons que ce terme est généralement réservé en ancien français aux textes sacrés ou exégétiques. L'assimilation du récit fictionnel à une escripture (et non à un conte, un roman, une estoire, un sermon ou autre) témoigne d'un désir, poussé à l'extrême, de sacraliser le récit.

26. Voir également, entre beaucoup d'autres exemples possibles, l'idéal poétique sous-jacent au prologue du roman Guillaume d'Angleterre: «Ja autre garde n'i prendra: La plus droite voie tandra,/ Que il onques porra tenir/ Si tost puisse a fin venir » (v. 7-10).

27. In Les CEuvres d'Adenet le Roi (1996). Gautier D'Arras évoque, quant à lui, dans son roman Ile et Galeron, le principe de la tempérance, la sobriété et la pondération devant être la mesure du discours poétique jugé vrai: "atempreüre/ Voel metre en ceste troveüre/ Et trover atempreement (...)» (v. 5-7). D’où le fait que les prologues insistent si souvent sur la rhétorique de la brièveté (brevitas), le processus d'amplification (amplificatio) impliquant l'idée de déformation, de mensonge ou de déviance que le Roman du roi Yder condamnait dans l'hyperbole.

28. Prise également ici au sens rhétorique du terme comme "narratio rei gestae» où la connaissance suppose une continuité et une quasi simultanéité entre le témoignage de visu des faits et le passage à l'écrit : «Dicta autem Graece historia APO TOU ISTOREIN, id est a videre vel cognoscere » (Isidore de Séville, Étymologies, I, XLI, 1).

29. Cette dimension testimoniale est particulièrement évidente dans le prologue de La Prise d'Orange, le poète incitant les pèlerins qui ont vu les armes-reliques des guerriers carolingiens sur les chemins de Saint-Gilles (Ami et Amile évoquera les pèlerins qui vont à Saint-Jacques de Compostelle) à valider sa version de l'estoire.

30. Réduire au maximum l'écart entre le temps de l'histoire et le temps de l'écriture est ici évidemment un moyen d'accroitre l'effet de réel et de vérité.

31. Seul le $m s c$ donne cette leçon du texte, ce qui est très révélateur quant à la nature potentiellement sulfureuse du terme. Le manuscrit D55 de la Bibliothèque ambrosienne de Milan suivie par E. Baumgartner et F. Vielliard donne « la continue » que le copiste a sans doute trouvée plus conforme et cohérente avec une poétique de la continuation sur laquelle se fonde en grande partie de roman antique.

32. "Crestïens se viaut antremestre,/ Sans riens oster et sans riens mestre,/ De conter un conte par rime/ Ou consonant ou lionime,/ Aussin com par ci lou me taillie,/ Mais que par lou conte s'an aillie ;/ Ja autre garde n'i prandra,/ La plus droite voie tendra/ Qu'il onques la puisse tenir,/ Si que tost puisse a fin venir./ Qui les estoires d'Angleterre/ Voudroit ancerchier et anquierre/ Une qui molt feit bien a croirre,/ Por ce que plaissant est et voire,/ An troveroit a Seint Esmont ;/ Se nus m'an demande le non,/ La l'aillie queirre se il viaut » (v. 1-17).

33. Ce renversement total est extrêmement intéressant comme si l'écrit - associé à la dimension symbolique de la Loi, ne pouvait autoriser l'excès déstructurant du désir transmis par ce récit.

34. La dimension du secret en rapport à la clôture, que nous avons signalée plus haut, est ici renforcée par la nature « oscurs » $(16,101)$ de l'auteur même du livre.

35. « (...) 'et je te dirai tel chose que nus hom, fors Dieu et moi, ne te porroit dire. Si en fai un livre (...). Or quier encre et parchemin adés, car je te dirai maintes choses que tu ne cuideroies que nus hom poïst dire'« $(16,37-39 ; 58-61)$; « 'Lors si assembleras ton livre au lor [le Joseph], si sera bone chose provee de ma poine et de la toue (...). Et quant li dui livre seront assamblé, s'en i avra .I. biau, et li dui seront une meisme chose, fors tant que je ne puis pas dire ne retraire, ne droiz 
n'est, les privees paroles de Joseph et de Jhesu Crist.' Einsi dist mes sires Robertz de Borron qui cest conte retrait que il se redouble, et einsi le dita Mellins, que il ne po savoir le conte dou Graal » (17, 108-116).

36. "'Mais il ne sera pas [ton livre] en auctorité, por ce que tu n'ies pas ne ne puez estre des apostoles, car li apostole ne mistrent riens en escrit de Nostre Seingnor qu'il n'eussent veü et oï et tu n'i mez rien que tu en aies veu ne oï, se ce non que je te retrai' « $(16,96-101)$.

37. Idée que l'on retrouve également clairement exprimée dans le prologue des Lais de Marie de France.

38. «Par lius est mençungiers e senz pleneireté ;/ E nepurquant i a le plus de verité » (v. 156-157).

39. Ne reconnaissons-là le titre de cet important essai de P. Zumthor (1987) qui a profondément marqué et renouvelé les études médiévales.

\section{RÉSUMÉS}

Si l'émergence du roman (comme langue et discours poétique) au Moyen Âge témoigne d'une émancipation face aux modèles latins et néo-latins qui implique une conscience aigüe d'une dynamique scripturaire agissant au cœur de la fiction, les réflexions menées dans les années 80 par Paul Zumthor et Bernard Cerquiglini ont bien montré que cette littérature naissante ne suppose nullement une rupture face à la matrice orale sur laquelle elle se fonde. Mais l'inverse est tout aussi vrai, les prologues nous rappelant constamment que la légitimité du fait littéraire ne saurait se fonder entièrement sur une éthique de la voix, mais qu'elle suppose de plus en plus une épistémologie de l'écrit dont le texte exhume les vestiges, réels ou fictionnels. Le développement d'une conscience littéraire en langue vulgaire n'est donc ni simple affirmation d'une présence vocale qui résonne au creux de l'écriture, ni pure revendication du pouvoir de la lettre face à une oralité dont le prestige symbolique commence à s'émietter au XII ${ }^{\mathrm{e}}$ siècle : comme le montrent les récits, la fiction se définit surtout comme pensée et expérience de l'entre-deux qui émerge de la tension constante, voire des contredictions ( $R$. Dragonetti), entre la lettre et la voix dont les frontières instables et fluides dessinent une poétique de l'incertitude renvoyant très souvent le lecteur au non-lieu d'une impossible confrontation.

The emergence of the romance (as language and poetic discourse) in the Middle Ages shows an emancipation from Latin and neo-Latin literary models that suggests an acute perception of the scriptural dynamic acting at the heart of the fiction. Nevertheless, Paul Zumthor's and Bernard Cerquiglini's works in the 1980s have clearly shown that this emerging literature does not suppose an interruption with oral background on which it is based. But the opposite is equally true: the prologues remind us constantly that the legitimacy of the literary phenomenon cannot be based entirely on an ethics of the voice and that it supposes more and more an epistemology of the writing. The development of a literary consciousness in the vernacular language is thus neither simple affirmation of a vocal presence that deeply resonates in the texture of the written poems, nor a pure claim of the power of the letter that threatens the fragility of the orality whose symbolic prestige begins to decline especially from the 12th century: as romances and chanson de geste show us fiction seems to appear above all an experience of the in-between which emerges from the constant tension, even counter-diction (R. Dragonetti), between the letter and the voice. 
INDEX

Mots-clés : littérature française, Moyen Âge, oral/écrit, fiction, poétique de la voix

Keywords : french Literature, Middle Ages, orality/writing, fiction, poetics of the voice

\section{AUTEUR}

CARLOS F. CLAMOTE CARRETO

Universidade Nova de Lisboa | IELT

ccarreto[at]@fcsh.unl.pt 